\title{
EL OJO DEL PODER EN EL ESPACIO DEL SABER: LOS INSTITUTOS DE BIOTIPOLOGÍA ${ }^{1}$
}

\author{
Gustavo Vallejo
}

CONICET, La Plata, Argentina

\section{RESUMEN}

Desde que en 1922 el médico endocrinólogo Nicola Pende lanzó su Biotipología, al tiempo que se precipitaban los acontecimientos políticos que derivaron en la «Marcha sobre Roma», la eugenesia italiana entabló una simbiótica relación con el poder al que nutrió "científicamente" de fuertes aportaciones en materia racial. La propuesta de Pende que confería un papel central a Institutos que habrían de establecer patrones de normalidad racial por medio de exámenes a toda la población, también se expandió internacionalmente encontrando una notable receptividad en la Argentina, donde su desarrollo trascendió largamente en el tiempo a la interrupción que sufriera en Italia tras producirse la caída del fascismo. Abordando esa problemática el pretende adentrarse en la historia de las interacciones mantenidas entre ciencia y poder, iluminando momentos en los que alcanzó una inusitada intensidad, revelada y reforzada a través de la cultura material.

PALABRAS CLAVE: Argentina, biotipología, eugenesia, Nicola Pende.

\section{SUMMARY}

From 1922, moment when the endocrinologist Nicola Pende wrote his Biotipología - at the same time of the «March over Rome»— the Italian eugenics began a symbiotic relation with the politic power, whose was scientifically full of its racial considerations. Parallel, Pende's theory —and the central role gave to Institutes that would establish racial normality patterns though examinations to every peoplespread internationally, too. It founded notable receptivity in Argentina, where its development lasted longer the end of fascism. So, the aim of this paper is to penetrate in the story of interactions between science and power, lighting their more intensives moments by means of material culture.

KEY WORDS: Argentina, biotypology, eugenics, Nicola Pende.

1 Trabajo realizado en el marco del proyecto «Biología, evolucionismo y eugenesia. Teoría y práctica en España y Argentina» financiado por el MCyT de España (BHA 2002-00588). 


\section{De LA EUGENESIA A LA BIOTIPOLOGÍA}

Tras originarse en el ultraliberalismo británico que a fines del siglo XIX leyó la darwiniana struggle for life en términos sociopolíticos, la eugenesia alcanzó una rápida difusión universal adquiriendo a su vez particulares características en los puntos de recepción de este discurso científico. En efecto, su articulación en tiempo y espacio, con singulares climas de época y con las diferenciadas formas en que cada nación moderna fue construyendo su propia noción de ciudadanía en tanto arena de negociación de deberes y derechos entre estado y sociedad-, abren un amplio campo de alternativas, aunque ellas nunca dejen de remitir al carácter coercitivo de la matriz originaria.

Un momento clave en la propagación de la eugenesia lo constituyó la decepcionante realidad que vivía Europa tras quedar devastada por la «gran guerra». Ese contexto resultó particularmente propicio para que la ciencia creada por Francis Galton acompañara la reconversión del ultraliberalismo en estados totalitarios basados en una competencia entablada no ya entre individuos sino entre corporaciones.

Mientras por entonces el decadentismo spengleriano constituía la más difundida interpretación de la crisis europea a la que asimiló biológicamente al natural declive que experimenta un organismo vital tras alcanzar su plenitud, la eugenesia rápidamente fue abriéndose con su promesa regeneradora. El inicio de la decadencia que Spengler situó en el momento mismo en el que la modernidad le restó a Occidente sus impulsos metafísicos, pudo entonces ser releído desde la eugenesia a partir de la directa atribución a cuestiones como la «degeneración» de la raza blanca por mestizajes inapropiados o el apartamiento a cumplir con el ineludible mandato que le imponían sus tradiciones culturales.

Dentro de las distintas alternativas eugénicas que la Europa de la primera posguerra fue cultivando, la italiana adquirió fuertes particularidades que se vinculan a su capacidad articuladora de experiencias internacionales que podían compartir un mismo mito de origen signado por la «latinidad». Así, tras la «gran guerra», Italia gestó por intermedio del médico endocrinólogo, Nicola Pende, la biotipología como disciplina encargada de llevar a cabo en el mundo latino lo que sería entendido como una instrumentación «práctica» de la eugenesia galtoniana. La creación científica de Pende iluminaría desde la década del 20 la nueva realidad política suscitada en Italia tras la «marcha sobre Roma», pero también la de otros países «latinos» como España y especialmente la Argentina.

El eje de esta nueva dimensión asignada a la Eugenesia, estaba dado por la invención de un dispositivo físico-institucional emergente de la esfera del saber: el Instituto de Biotipología. Consistía en un verdadero observatorio de todos los 
comportamientos humanos situado en un ámbito capaz de ejercer coacciones a través del espacio y del protagonismo de la inquisidora mirada científica. Ya no se trataba del tradicional procedimiento higienista de aislar lo «anormal» para encerrarlo en sitios que dejaran a salvo al universo de la «normalidad», sino que propendía a que los institutos fueran jerarquizados puntos de vigilancia dirigidos a todos los individuos. Para ello avanzaba hacia la conformación de un programa que contenía el germen de una homogénea distribución espacial de estos establecimientos científicos concebidos para ejercer un estricto control social y a los que Nicola Pende imaginó extendidos capilarmente sobre un vasto territorio, primero nacional y después internacional.

El paso de la teoría galtoniana a la biotipología, estaba entonces signado por aquella voluntad de acentuar la «aplicación práctica» de la eugenesia, que Pende vislumbró a través del énfasis puesto en un anclaje físico-intitucional que incluía su taylorista reproductividad ${ }^{2}$. Y precisamente en este aspecto, su teoría reinstaló de otra invención física-institucional como era el panóptico de Bentham los propósitos de conseguir que un espacio de características reproducibles permita controlar una multitud a través de una única mirada ${ }^{3}$. La biotipología modernizó al panóptico, profundizado los alcances de un espacio de vigilancia para cárceles, hospitales y escuelas en el que poder se ejercía por transparencia y el sometimiento se obtenía por la proyección de claridad ${ }^{4}$. En la biotipología la transparencia se obtenía de los diversos estudios sobre el cuerpo y el alma a los que era sometido cada individuo dentro de un espacio del saber y la proyección de claridad la aportaba el incuestionable diagnóstico científico.

Con el nuevo descubrimiento de Pende nacía entonces un mecanismo que tenía su fundamento en la detección de anormalidades físicas, psíquicas y morales no visibles que anticipen la comisión de actos perturbadores el orden público. En esta economía del poder era fundamental reconocer al «otro», aquel que se apartaba de la «normalidad», antes que la manifestación pública de ese apartamiento obligue al estado a

2 Si bien el Palacio de Homicultura proyectado en Cuba 1910 podría considerarse como un antecedente válido, las valencias atribuidas por los eugenistas cubanos Ramos y Hernández a lo que debía ser el único y gran monumento para la ciencia de la raza, lo distancian del taylorista programa pendeano. Sobre el proyecto cubano véase GARCÍA GONZÁLEZ, A. y ÁlVAREZ PelÁEZ, R. (1999), «El Palacio de Homicultura»; en En busca de la raza perfecta, Madrid, CSIC, pp. 121-132.

3 Foucault, M. (1979), «El ojo del poder»; en Bentham, J. (1979), El Panóptico, Madrid, Ediciones de La Piqueta.

4 El panóptico consistía en un edificio circular dividido en celdas con ventanas al exteriior, por donde entraba la luz, y ventanas al interior, donde una torre central en la que se hallaba el vigilante percibía a través de la claridad proyectada la silueta de los individuos encerrados. 
llevar a cabo mas costosas acciones represivas 5 . Para ello debía detectarse la «otredad» en la esfera privada y mas aún en lo mas íntimo que encerraba cada ser, a través de la información que podía proporcionar la ciencia de la constitución humana y el confesionalismo religioso. Vale decir que con la biotipología toda la población debía quedar bajo la atenta mirada de una ciencia concebida para identificar y aislar aquello que en última instancia podía poner en riesgo la gobernabilidad.

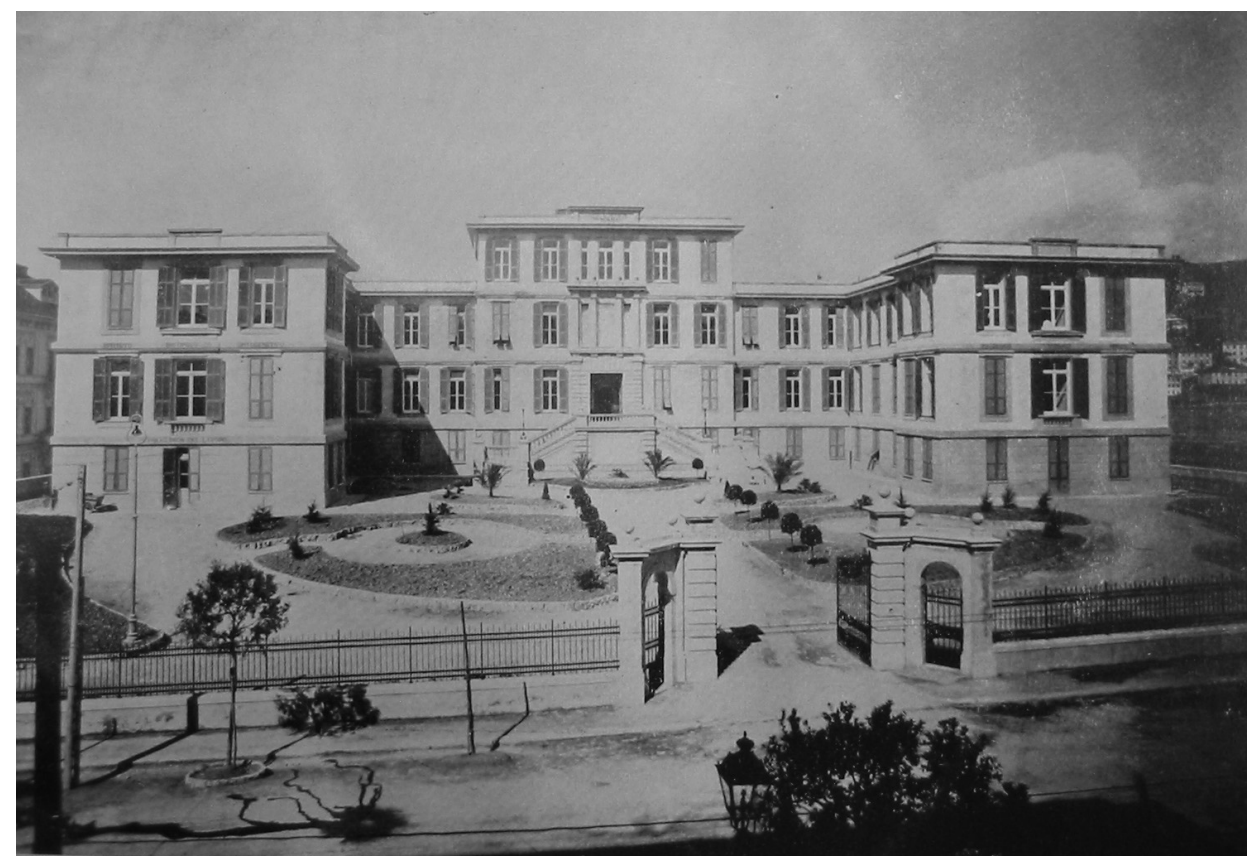

Instituto Biotipológico Ortogenético de Génova.

Quien vigilaba, esto es aquel que para Bentham podía hacerlo por ser el más «confiable» al poder era, en la nueva realidad italiana, el científico aliado a la fe

5 Foucault señala que el problema del panóptico apunta directamente al precio del poder. El poder «no se ejerce sin gastos. Existe evidentemente el costo económico». «Pero además está el costo propiamente político. Si se es muy violento se corre el riesgo de suscitar insurrecciones; si se interviene de forma discontinua se arriesga uno a dejar que se produzcan en los intervalos fenómenos de resistencia de un coste político elevado». FoUCAULT, M. (1979), p. 17. 
católica y sus instrumentos para llegar a la verdad, para mirar a todos avanzando más allá de las evidencias, de los comportamientos externos expresados en su apariencia y en sus actos, quedaban resumidos en una ciencia que ponía en duda por principio la tradicional oposición a la forma de distinguir lo normal de lo patológico a través de los caracteres externos. No es que el pensamiento de Cesare Lombroso fuera cuestionado en su afán patologizador de quien por poseer determinados caracteres fenotípicos fatalmente habría de apartarse en algún momento de la normalidad 6 , sino que ahora, imbuido del pensamiento tomista Pende trataba de valerse de nuevos instrumentos de medición física y moral para complejizar y profundizar los mecanismos de detección de lo anormal que había creado la criminología positivista. La ciencia de Pende, entonces, introduciéndose en lo profundo del ser a través de un desplazamiento de la antropología física a la antropología endócrina, que era inclusivo a su vez de los aportes que le proveía la unidad sustancial entre cuerpo y alma sostenida por el pensamiento aristotélico-tomista, nació para detectar «alteraciones individuales» de tipo hormonal y moral capaces de transmitirse a la esfera social. Con ese fin llevó la preocupación de Lombroso por estudiar las «disgenesias» de quienes poseían un visible apartamiento de la normalidad hasta límites insospechados. Para llegar a la verdad que podían ocultar las apariencias, hacía falta analizar particularizadamente a cada uno de los integrantes de toda la población, desenmarañando los infinitos obstáculos que interferían ese propósito cuando un cuerpo sano escondía un alma criminal.

La biotipología acentuaba aquella confluencia entre poder y saber que el panóptico puso de manifiesto para materializar la ilusión de hacer visible, de transparentar lo más íntimo del individuo, lo que ocultaban los cuerpos, llegando en definitiva a encontrar lo patológico en lo profundo del alma. Ello fue posible cuando quedaron integrados los materiales provenientes de la crimonología positivista a la antigua conjunción premoderna entre medicina y religión que dio origen a los primeros establecimientos hospitalarios europeos, resultando de ello un dispositivo articulador de todas las variables necesarias para poner en funcionamiento lo que llegó a pensarse como el más infalible mecanismo de detección de lo «anormal». De este modo, la biotipología pendeana logró ampliar el espectro de adhesiones suscitadas por la matriz galtoniana para capitalizar la confluencia de liberales que desde su deslumbramiento inicial por el darwinismo social llegaban a la eugenesia y de católicos integristas reticentes a aceptar toda expresión que tuviera algún sesgo positivista pero deseosos de instalar políticas de control social. Ello fue posible gracias a la

6 Para conocer en profundidad la producción de Cesare Lombroso es indispensable ver la obra de PESET, J. L. y PESET, M. (1975), Lombroso y la escuela positivista italiana, Madrid, CSIC. 
hábil manipulación de un discurso científico que tendía a relativizar el fundamento darwiniano y sus reelaboraciones italianas llevadas a cabo por Lombroso, que dio lugar a una curiosa derivación metafísica de la biología capaz de comprometer firmemente en ella a figuras tan influyentes como el padre Agostino Gemelli, rector de la Universidad Católica de Milán, presidente de la Academia Pontificia y a la postre el principal enlace entre el régimen fascista y el Vaticano ${ }^{7}$.

Y del mismo modo que lo hiciera Bentham con su invento, Pende ofreció explícitamente su ciencia al poder, asegurándole resultados de sumo interés que trascendían las esferas particularizadas del médico, del higienista, del educador, del antropólogo y del biólogo, aunque también esas esferas quedaran involucradas. El hecho de que este nuevo saber se fundara en acceder al conocimiento de los hombres en el cuerpo y en la mente, para valorarlos, mejorarlos y utilizarlos en pos del máximo rendimiento de la colectividad, lo volvía central para la dirigencia política. Y si en 1922 estas ideas ya conformaban el corpus de su doctrina contenida en La biotipologia umana, en la que proporcionaba al poder la fórmula constitucional completa, somática y psíquica de cada individuo en un registro capaz de condensar el «biotipograma individual», en tanto «registro personal de la salud y de la individualidad», en los años siguientes esa subsidiariedad de su saber al poder se plasmó en una acción conjunta. Para el penalista y eugenista español, Mariano Ruiz Funes, la biotipología ya era a fines de los años 20 un cualificado «punto de intersección entre la doctrina positiva y el evangelio de Mussolini» ${ }^{8}$. Algo que el propio Pende enfatizaba al describir su creación como un saber de utilidad para quienes «estudian los problemas de la herencia y los del mejoramiento de la raza», ya sea el criminalista filantrópico «que anhela la redención de los inmorales y de los candidatos al delito», o el filósofo que persigue la «eterna cuestión de las relaciones entre personalidad física y personalidad psíquica». Y fundamentalmente para que «el hombre político y el director de pueblos, logre la instauración de una política nueva», que debía llamarse «política biológica»?.

7 La trayectoria seguida por Gemelli tiene puntos de confluencia con la de Enrico Ferri. $\mathrm{Al}$ igual que este tuvo una primera etapa socialista. Para exaltar su apartamiento de ese pasado Gemelli cambió su verdadero nombre de Eduardo por el de Agostino, aludiendo así directamente a la historia de San Agustín signada por una vida licenciosa que una revelación divina modificó drásticamente tras instarlo a que la consagrara a Dios.

8 Ruiz FunES, M. (1929), Endocrinología y criminalidad, Madrid, Morata, p. 316.

9 PENDE, N. (1932), Trabajos recientes sobre endocrinología y psicología criminal, Madrid, Javier Morata, p. 86. Esta traducción al español con prólogo de Mariano Ruiz Funes sólo demoró unos meses de la original edición italiana de mayo de 1932. 


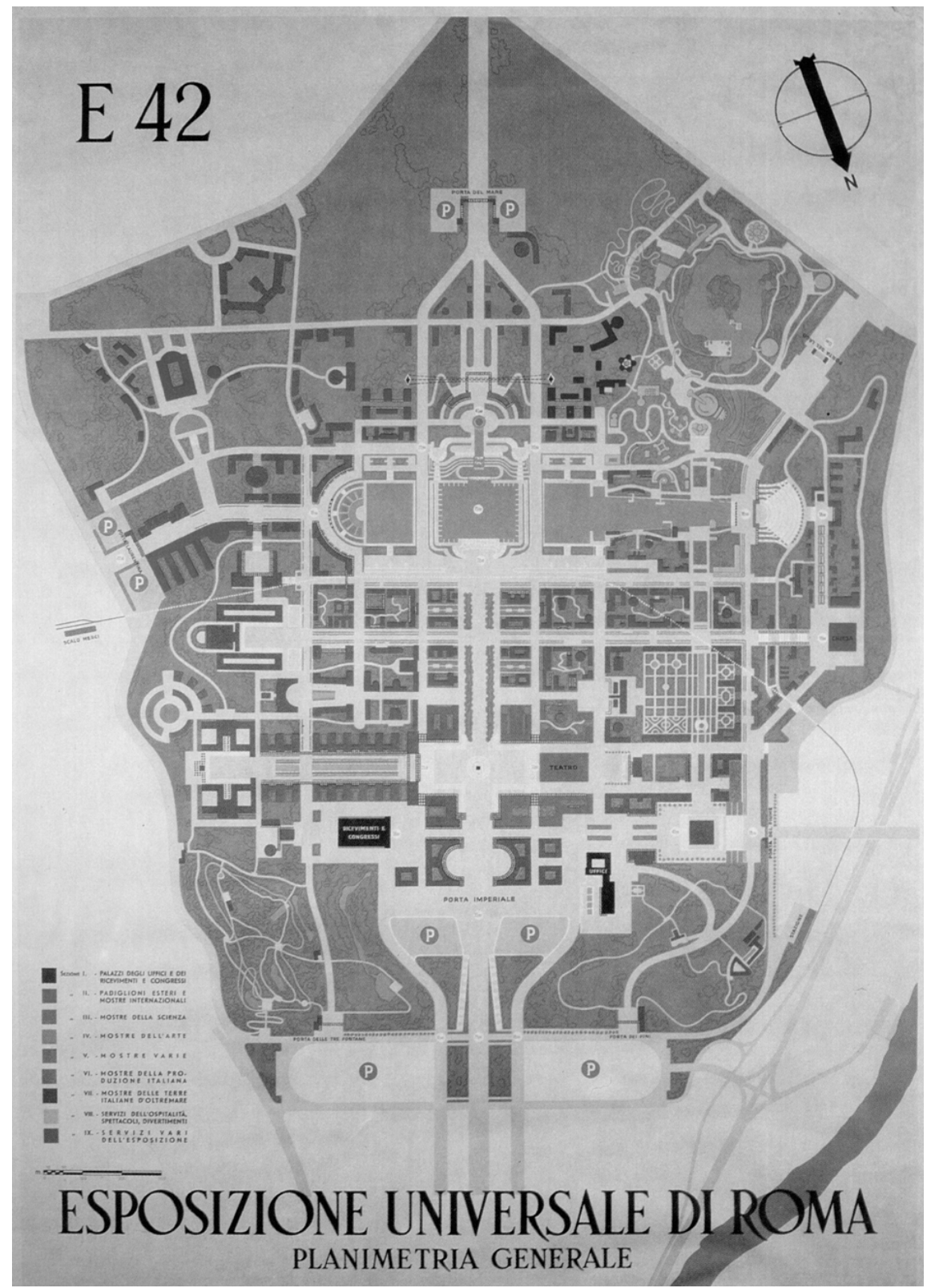

E42. Planta general. Véase arriba a la izquierda el instituto de Bonificación Humana y Ortogénesis de la Raza. 


\section{El INSTITUTO BIOTIPOLÓGICO ORTOGENÉTICO DE GÉNOVA}

A comienzos de la década del 20, Pende ya había desarrollado suficientemente sus ideas en la Universidad de Génova como para explicitarlas ante un nuevo poder político particularmente predispuesto a oirlas. Si bien la relación de Pende con el poder se había visto favorecida años atrás cuando su pericia profesional había servido para curar a la hija de Vitorio Emanuele III de la anorexia que padecía, con el ascenso de Benito Mussolini sus ideas encontraron inusitadas posibilidades de canalización. En efecto, en 1925 su biotipología recibió la explicitación pública del reconocimiento del estado, cuando al mismo tiempo que el Duce quedaba consagrado oficialmente como dictador de Italia, éste se decidía iniciar la construcción en la Universidad de Génova de un establecimiento modélico para dar comienzo a la propagación capilar de una red de dispositivos similares en todo el territorio italiano.

La biotipología llevaba a cabo el análisis de la personalidad humana, evaluando el «capital humano» y aquellos valores somáticos, morales e intelectuales que más preocupaban a quienes para Pende eran «los clarividentes rectores de la nueva Italia» ${ }^{10}$. Sus aportes así se vieron desde un principio inescindiblemente ligados a las acciones gubernamentales de un régimen interesado en obtener las respuestas deseadas en la sociedad reduciendo los costos de sus intensas intervenciones directas. La rigurosa observación y evaluación de toda la población no sólo podía facilitar una tarea preventiva ante lo insumiso, sino también favorecía la «regeneración» de seres proclives a caer en conductas desviadas de la normalidad antes de que ellas se manifestaran.

En el programa de Pende confluyeron otros importantes antecedentes italianos, como eran los estudios de la personalidad humana individual que se remontaban a los trabajos que cinco décadas atrás había iniciado Achille De Giovanni y proseguido con un sentido más poliédrico Giacinto Viola, padre del constitucionalismo. Claro está que además de ellos también la figura de Cesare Lombroso y su «antropología criminal» sobrevolaba en la formación de los integrantes de la nueva experiencia científica pendeana.

Dos destacados colaboradores de Pende, Giuseppe Vidoni y el sacerdote Mario Barbara describieron la experiencia como «una clínica donde los supuestamente sanos podían científicamente ser examinados con los mas perfectos medios de indagación médica moderna a fin de develar diversas predisposiciones morbosas, hereditarias o adquiridas y los temperamentos morbosos y submorbosos, que ponen en evidencia aquella serie infinita de anomalías y anormalidades,

$10 \quad$ Ibidem, pp. 115-117. 
de debilidades y errores de la constitución del cuerpo y de aquella mente existentes o en estado latente». Predisposiciones que «a modo de enemigos internos, insidiosamente se ocultan en los escondrijos de nuestros órganos y en nuestra sangre, amenazando la salud y a menudo la existencia misma» ${ }^{11}$. Se trataba entonces de una «clínica de sanos», que asignaba particular importancia a la examinación de distintos grupos sociales. En especial a los adolescentes, futuros ciudadanos en los que debían detectarse las capacidades de acuerdo a la organización científica del trabajo establecida por el estado fascista y los obreros, de quienes los industriales que sostenían el régimen esperaban una escrupulosa evaluación que permita seleccionarlos de acuerdo al estado físico y su disciplinamiento.

«Muchos y buenos, fuertes de número, pero también de cabeza, de corazón y de músculos» ${ }^{12}$, esa era la meta ideal a la que apuntaban los trabajos del instituto de Biotipología. Inmerso en un claro «poblacionismo selectivo» que en adelante iría caracterizando a quienes desde países como Italia, España y Argentina abrazaron los ideales de esta eugenesia desplegada en el mundo latino ${ }^{13}$, quedaba en claro que la tradicional «medicina social» no podía atender «el número rápidamente creciente de la población, sin preocuparse también de la buena calidad del gérmen de la Italia futura ${ }^{14}$. Esa medicina social por si sola resultaba insuficiente si no era entendida como un componente más dentro de este vasto programa que trascendía largamente sus alcances. La biotipología que la englobaba, no constituía solo una ciencia de dominio exclusivo del médico ni estaba dirigida solo a las generaciones presentes, sino que se lanzaba directamente a la esfera política para proveerla de una profunda información social que tras ser recabada desde la biología y la psicología tomista quedaba al servicio del modelado de la raza futura.

«Biología política o política sobre bases biológicas», el programa ideado en esos términos por Pende para ser llevado a cabo a través de los institutos biotipológicos constaba de seis secciones: Sección de medicina y de higiene individualizada, que era un centro de observaciones periódicas de los «normales» y de «curación de los débiles de cuerpo y de mente». Sección de biología de la raza y de eugenesia, ocupada del «estudio de la herencia, para la higiene pre-matrimonial y pre-natal».

11 Barbara, M. y Vidoni, G. (1933), L'Instituto Biotipológico Ortogenético di Genova, Genova, Carlo Badiali e C., p. 5-6.

12 Ibidem, p. 11.

13 El concepto de «poblacionismo selectivo» es utilizado por Marisa Miranda para describir las políticas pro-natalistas de impronta eugénica que tuvieron una particular afinidad en la Italia fascista, la España franquista y la Argentina de 1930 a 1983. Véase MIRANDA, M., «La Biotipología en el pro-natalismo argentino (1930-1983)», Asclepio, en prensa.

14 BARbara, M. y VidOni, G. (1933), p. 11. 
Sección de pedagogía, organizada «sobre bases ortogenéticas, constitucionalísticas», donde médicos y psicólogos formaban los «registros de la personalidad» del escolar. Sección de antropopsicología criminal, diseñada para la «valoración médico-jurídica, justa y moderna, del delincuente y para la utilización de los inmorales y de los amorales». Sección de orientación y de selección profesional y de taylorismo, gestada para la «preparación científica de los trabajadores», teniendo como principal finalidad lograr «una selección y una valoración racional de los dedicados a los varios oficios y profesiones estatales». Sección de política biológica, pensada para la «organización del Estado sobre bases bio-psicológicas, naturales y seguras, originarias de la verdadera competencia y de las diferentes necesidades de las varias categorías de individuos ${ }^{15}$.

Y si inmediatamente después de que Pende lanzara su programa ideal, éste podía encontrarse descripto con bastante precisión en La Semana Médica, el principal órgano de corporación médica argentina, ello venía a confirmar que cuanto menos entre ambas naciones ya estaban afianzados los mecanismos de circulación de ideas a partir de los cuales podía avanzarse hacia la conformación de la red de la eugenesia latina. Pero además del interés por dar cuenta inmediatamente de un proyecto para el desarrollo de la biotipología italiana, la voluntad de integración a esa naciente eugenesia latina era palpable en la imperatividad con la que influyentes médicos argentinos solicitaban anticiparse incluso a Italia en la tarea misional de instalar esas verdaderas máquinas del poder para el control de la raza. Siendo para ellos un mandato de toda nación civilizada poseer institutos de este tipo, se hacía «impostergable» su aplicación en este país para estudiar «el crecimiento normal, lo anómalo y lo patológico». Estos propósitos «debían» canalizarse en la forma que preveía Pende atendiendo a las «peculiares condiciones de ambiente étnico-social, en la seguridad de que, con su realización, se habría logrado dar el primer decisivo paso en el sentido del perfeccionamiento físico y moral de la raza» ${ }^{16}$.

Pero a pesar de los esfuerzos argentinos por disponer de esa novedad de la ciencia del control social, fue lógicamente en Italia donde las ideas de Pende encontraron su inicial concreción. El 7 de diciembre de 1926 el ministro de instrucción pública Pietro Fedele, inauguró el primer instituto de Biotipología en Génova, colocando a esta iniciativa en «correspondencia plena con la dirección adoptada por el gobierno fascista» en materia racial. Así, «el gran movimiento iniciado en toda la nación para el mejoramiento de la raza, para la protección de

15 «Eugenética», La Semana Médica, no 45, Buenos Aires, 8 de noviembre de 1923, pp. 1037-1038.

16 Ibidem, p. 1037. 
la salud, para la higiene mental alcanzaba en Genova su máxima expresión», al decir de Vidoni y Barbara ${ }^{17}$.

Presentado ante la sociedad como un «Templo dedicado al arte de la ciencia médica y al sentimiento de humanidad $»^{18}$, el instituto fue enclavado en la colina de Álbaro, en las inmediaciones del viejo hospital San Martino, atendiendo a los deseos de Pende de distanciarse de la realidad urbana a la que a su vez podía controlar visualmente desde su estratégica ubicación en el punto mas alto de la región.

En su interior, inequívocos signos afianzaron los objetivos perseguidos. Mientras un esquema típicamente hospitalario permitía distribuir las salas de los sucesivos exámenes a ambos lados de un amplio corredor, una sala central condensaba expresamente la dirección y finalidades del Instituto que quedaban resumidas en la figura vigilante de un gran retrato del Duce junto a la siguiente frase grabada: «Conócete a ti mismo y a los otros. Solo así podrás comprenderte y comprender. Mejorarte y mejorar. Dominarte y dominar ${ }^{19}$.

Otra sala del instituto albergaba imágenes que buscaban dar cuenta del directo paralelismo que la teoría de Pende asignaba a la perduración de la concepción clásica de la medicina en sus innovaciones científicas. Junto a la reproducción del cuadro de Alberto Durero (1471-1528) que representaba los cuatro temperamentos concordantes con los cuatro cuerpos simples (agua, aire, fuego, tierra) y de las cuatro propiedades fundamentales (húmedo, seco, caliente, frío), se situaban las cuatro variedades biotipológicas (dinamico-humoral) individualizadas por Pende. La biotipología era entonces la síntesis de ambas representaciones, la de los cuatro temperamentos de la antigüedad y la de los cuatro biotipos de la escuela genovesa.

La insistencia en la cuestión numérica remitía a su vez a la ley pitagórica del número y de la armonía, en tanto canon matemático invocado para explicar las proporciones recíprocas que dan belleza ideal al cuerpo y sus correlaciones armónicas de las que resulta del estado óptimo de salud. Y si en Pitágoras Pende identificaba los orígenes de la ciencia latina que fluían en su ideal racial debido a la escuela fundada por aquel en Crotone, la verdadera obsesión asignada al número cuatro se trasladó a una ampliación de la matriz científica que sumó a los cuatro biotipos la teoría de las cuatro armonías biológicas que debían perseguirse para alcanzar la eugénica perfección humana: la belleza que era la armonía de las formas; la salud que era la armonía de las funciones; la bondad que era la armonía de los sentimientos; y la sabiduría que era la armonía del intelecto. La síntesis gráfica de esta enunciación estaba dada por un cuadrado cuyas cuatro caras ten-

17 BARBARA y VidONI (1933), p. 13.

18 Ibidem, p. 16.

19 Ibidem, p. 21. 
dían a confluir armónicamente, hecho que al producirse generaba la forma de una pirámide con su vértice que sintetizaba el punto culminante de la perfección humana, síntesis vital de todos los procesos biológicos.

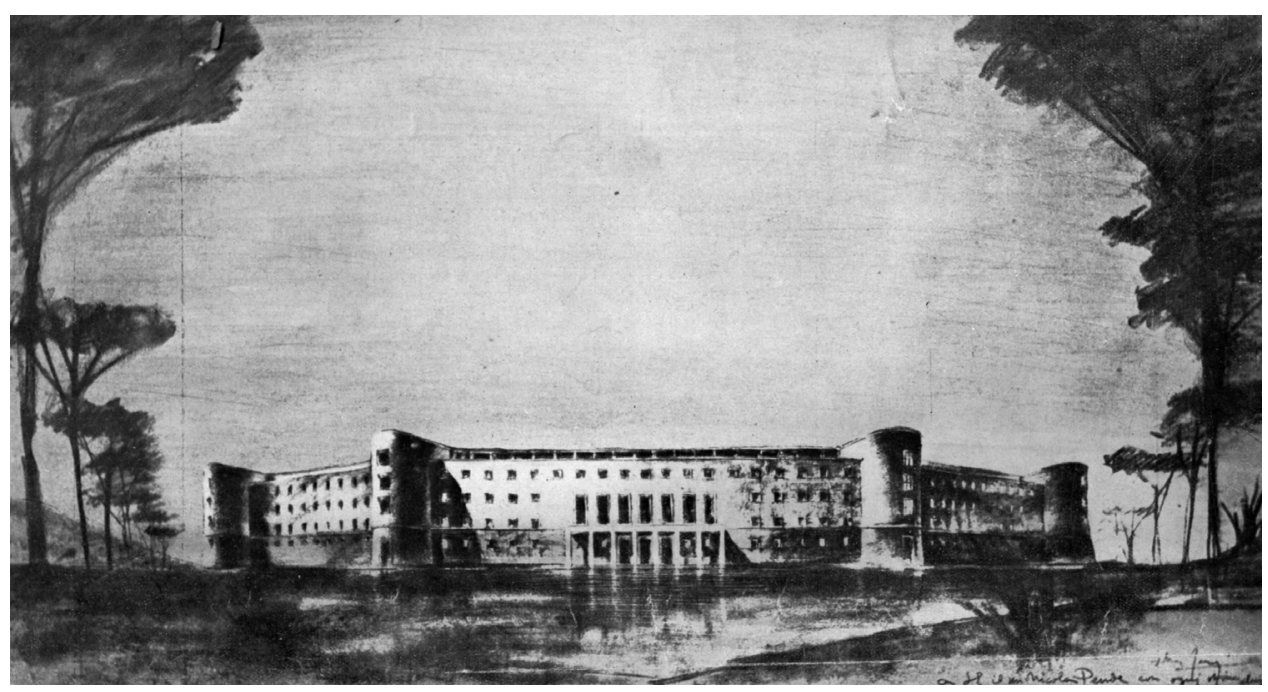

Instituto de Bonificación Humana y Ortogénesis de la Raza de Roma. Anteproyecto de su fachada publicado en Anales de la Asociación Argentina de Eugenesia, Biotipología y Medicina Social.

Sobre las actividades desarrolladas en el Instituto de Pende, se interesó especialmente Enrico Ferri, cuando, tras su etapa socialista se consustanció con los ideales del régimen fascista ${ }^{20}$. Y si entre médicos argentinos existía una inoculta-

20 Las reflexiones de Ferri luego de un atentado perpetrado contra Mussolini son elocuentes de su nuevo ideario: «Le dije (a la culpable) que Italia, desangrada en cuatro años de guerra, había caído en el bolchevismo más estúpidamente anárquico, porque carecía de programa: era un bolchevismo sin Lenin... Italia había llegado así al borde del abismo... De manera que vio en Benito Mussolini y en el fascismo creado por él un providencial salvador y acogió con entusiasmo su dictadura... En realidad, Mussolini tuvo y tiene una idea fuerza; y es la supremacía del Estado sobre el individualismo, que la Revolución Francesa reivindicó en justicia contra la tiranía medieval, pero que a fines del siglo XIX y en este siglo XX ha llegado en muchos países al exceso de un liberalismo que resulta libertario y fundamentalmente anárquico. Y toda la obra política y social de Mussolini ha sido y es la enérgica, inflexible 
ble avidez por conocer los avances de la biotipología italiana para extender a este país sus alcances, la aguda descripción que Ferri hizo del funcionamiento del Instituto en el diario La Prensa de Buenos Aires, daba cuenta del interés que el tema despertaba más allá del público especializado en las ciencias médicas. Según el destacado informante, el joven que ingresaba era sometido a un «primer estudio» dirigido por el sacerdote Mario Barbara, quien estaba secundado por sus ayudantes, los doctores Muggia, Bufano y Antognetti, en el cual se ponderaban las medidas de su cuerpo en relación a la edad, utilizando para ello el «antropómetro de volante» diseñado por Giacinto Viola y aparatos fotográficos específicos. Un segundo estudio se encargaba de la constitución orgánica del individuo, mediante el examen químico de la sangre, para detectar la estructura y la función de las glándulas internas que para Pende constituían los fundamentos biológicos de toda personalidad humana. La evaluación orgánica era, a su vez, completada con una observación de las funciones biológicas de oxigenación (asimilación química) y circulación (pulsaciones del corazón). Luego del estudio del cuerpo humano, en sus formas y en sus funciones vitales, se pasaba al «estudio psicológico» del individuo en la sección dirigida por el profesor Ragazzi, también director del Instituto Médico Escolar de Génova. Posteriormente eran examinadas las formas más simples y fundamentales de la actividad psíquica del sujeto tales como la atención y la memoria, con el propósito de «revelar y precisar las aptitudes para el trabajo» facilitando con ello la racional detección de aptitudes para desempeñarse con éxito en un oficio o profesión por el bien del «individuo, la familia y la sociedad». A estas «Secciones fundamentales» — estudio orgánico y estudio psicológico- se agregaban las secciones de aplicación práctica, cuyas tres principales eran la de orientación «médico pedagógica»; la de «prevención social» y la de «terapéutica»»" En la primera se observaban las aptitudes de cada escolar en relación a la pedagogía, seleccionando a los escolares «anormales» y dirigiendo a los normales al género de estudios - técnico o literario - que más se adaptaba «a su personalidad fisiopsíquica». Una «libreta biográfica» acompañaba a cada examinado, proporcionando «información invalorable» para evaluar «su desarrollo físico y psíquico, los progresos y los atrasos de su carrera escolar, las diversas aptitudes para el trabajo y la diversa conducta y disciplina social para con el maestro, los compañeros, la familia». La sección de «prevención social» se ocupaba especialmente de los niños de

realización de esa idea-fuerza y ha vuelto a dar a Italia el orden y la disciplina social» (FERRI, E. (1927), «Una loca contra un dictador-Violet Gibson contra Benito Mussolini», La Prensa, Buenos Aires, 22 de marzo, p. 15).

21 Ferri, E. (1927), «Un establecimiento único en el mundo», La Prensa, Buenos Aires, 28 de mayo, p. 9. 
mala conducta y de los delincuentes, que eran enviados al Instituto por las autoridades policiales y judiciales. El director de esta sección era el profesor Bidón, un antropólogo criminalista de formación lombrosiana encargado de estudiar las relaciones entre las perturbaciones endocrinológicas y la criminalidad.

Completaba el equipamiento del Instituto salas especialmente preparadas para llevar a cabo «aplicaciones terapéuticas individuales y colectivas» ${ }^{22}$, a efectos de profundizar el estudio «constitucional» de la persona humana desde el punto de vista anatómico, bioquímico y psicológico. Todo ello dentro del enfoque «poliédrico» orientado por la higiene y la terapia individual orientados hacia la prevención social de las anomalías humanas, que instaba a efectuar «una cotidiana y metódica observación de escolares, conscriptos, obreros, pequeños anormales y delincuentes para el mejoramiento de la estirpe y para la prevención de las enfermedades sociales en la tríada física del alcoholismo, tuberculosis y avariosis, y en la tríada psíquica de demencia, suicidio y delito». Vale decir que la escuela, el taller y el ejército encontraban entonces en este instituto los órganos técnicos para la observación, la vigilancia y la tutela de la crianza orgánica y psíquica de las nuevas generaciones que necesitaba el régimen fascista ${ }^{23}$.

Toda la información recabada por los diversos exámenes que indagaban los rasgos genéticos, psicológicos, morales y físicos de individuos «aparentemente sanos», quedaba condensada en la cartilla o ficha biotipológica que creara Pende como instrumento de aplicación de la eugenesia para llevar a cabo en diferentes ámbitos la identificación y selección de las capacidades. El uso de la cartilla biotipológica en las escuelas populares, donde se desempeñaba Ragazzi, fue destacado por Ferri, para quien esa iniciativa del régimen posibilitaba «fijar — con los métodos técnicos de la pedagogía antropológica - los datos mas característicos de la personalidad física, moral, intelectual de cada alumno, y precisar sus tendencias y aptitudes a la conducta social al trabajo». Otra cartilla estaba dirigida especialmente a «los alumnos deficientes o anormales, no solo moral sino, sobre todo, moralmente (candidatos a la delincuencia)». Para Ferri entonces los exámenes del instituto de Pende como el control que a partir de las cartillas podía ejercerse en las escuelas, permitía tener a disposición del régimen «datos abundantes y seguros sobre la personalidad y peligrosidad de todo ciudadano, a causa de su propia con-

22 Allí se buscaba introducir en la sangre de los individuos sustancias que escaseaban en ella. Para ese fin se recurría al método de la «inhalación en seco» consistente en el encierro de 20 ó 30 niños en una sala hermética, una especie de cámara de gas, con el fin de que sea absorbido calcio, yodo u otra sustancia a través de «pulvículos invisibles» con los que se saturaba el aire de ese ambiente. Ibidem.

23 Ibidem. 
ducta irregular o delictuosa», reclamando sobre sí la necesidad de aplicar medidas preventivas o represivas ${ }^{24}$. De hecho el criminalista Palópoli, asoció directamente al espíritu del Código Penal que Ferri presentó en 1921 y con modificaciones del ministro de justicia Alfredo Rocco fue puesto en vigor en $1931^{25}$, con el instituto de Pende, y adhiriendo a esta orientación requirió la creación de nuevos establecimientos para obtener con métodos de investigación médica, antropológica y psicológica, una cartilla de identificación modificada periódicamente ${ }^{26}$.

Pero no era solo en Italia donde desde mediados de los años 20 existía un ambiente general particularmente favorable para llevar a cabo estas acciones. Para Ferri «América Latina y, dentro de ella, la República Argentina» constituían «un gran observatorio y un campo abierto para todas las innovaciones de la vida individual y social» especialmente para las que impulsaba Pende, convertido ya en un paradigma del pensamiento científico italiano. En la misma dirección apuntaba el artículo que el penalista español Mariano Ruiz Funes - directo interlocutor de Pende en su país-, escribió en el diario La Nación de Buenos Aires, requiriendo la creación de institutos biotipológicos «para la profilaxis de ciertas formas de criminalidad ${ }^{27}$. Favorecía la receptividad en Argentina de estos planteos la notable convergencia experimentada por diversos sectores consolidados en los años 20 como grupos por demás influyentes en el poder público. Además de la intensa prédica eugénica que circulaba dentro de la corporación médica argentina, cabe referirse a las aplicaciones "prácticas» de la antropología lombrosiana llevadas a cabo por Víctor Mercante en la educación, las iniciativas de Víctor Delfino quien tras representar a la Argentina en el primer Congreso Internacional de Eugenesia celebrado en 1912 en Londres ideó la Sociedad Eugénica Argentina, la actividad de la Liga de Profilaxis Social creada con fines eugénicos por el doctor Fernández Verano, el espacio que la Eugenesia encontró en la orientación claramente regresiva del Museo Social Argentino, y las leyes eugénicas promovidas por el doctor Leopoldo Bard — diputado radical y luego ministro en el segundo gobierno de Yrigoyen-, que comprendieron la ley de «Defensa de la raza» sancionada en 1925 .

24 FERri, E. (1933), Principios de derecho criminal, Madrid, Reus, p. 305 (1ª edición italiana de 1927).

25 Sobre el «Código Rocco», puede verse Del CURA, M. «Ciencia médica y ciencia penal en el fascismo italiano: el Código Rocco»; en Huertas, R. y OrTIZ, C. (ed.) (1998), Ciencia y Fascismo, Doce calles, Madrid, pp. 61-75

26 RUIZ FunES, M. (1929), p. 316.

27 RuIz FunES, M. (1928), «El problema psicológico de la culpa penal», en La Nación, Buenos Aires, 4 de octubre. 


\section{DE LA BIOTIPOLOGÍA A LA BONIFICACIÓN HUMANA}

La utopía pendeana de irradiar internacionalmente su teoría y sus establecimientos a través de una red eugénica, se vio alimentada por la receptividad que ese propósito tuvo en países como la Argentina. Si por el impacto del crac del 29 se interrumpió allí el aluvión inmigratorio que en gran medida había estimulado el inicial desarrollo de la eugenesia, en adelante los eugenistas argentinos encontraron en la teoría de Pende la posibilidad de llevar a cabo la vieja consigna de poblar la nación, pero ahora favoreciendo la descendencia de los «mejores» a través de una articulación entre «cantidad» y «calidad» que se desplazaba de la selección de inmigrantes ${ }^{28}$ a un pronatalismo excluyente de inspiración fascista que se prolongó durante las cinco décadas siguientes ${ }^{29}$.

Los ya existentes lazos biopolíticos entre Argentina y el fascismo italiano se acrecentaron inusitadamente después del golpe militar de setiembre de 1930 que llevó al poder a figuras explícitamente consustanciadas con el ideario político imperante en Italia y Alemania. En noviembre de ese año, luego de ofrecer conferencias en España, Pende llegó a la Argentina invitado por el Instituto Argentino de Cultura Itálica y por la cátedra de clínica médica de la Universidad de Buenos Aires que estaba a cargo del doctor Manuel Castex, prestigioso médico que solo unos meses mas tarde sería ungido rector de esa Universidad. Pende dictó en Buenos Aires un curso intensivo de perfeccionamiento en endocrinología, e inmediatamente después, los argentinos Octavio López y Arturo Rossi partieron con él a Italia para cumplir una misión oficial encomendada por el gobierno del general Uriburu, consistente en el análisis y el estudio comparativo de los avances internacionales en materia de eugenesia y medicina social, poniendo especial énfasis en profundizar el conocimiento del Instituto que Pende había creado en Génova.

A su regreso, Rossi promovió con éxito la creación de la Asociación Argentina de Biotipología, Eugenesia y Medicina Social, institución fuertemente imbricada con el poder público, de la que Mariano Castex fue su primer presidente y Pende su primer miembro honorario corresponsal. Asimismo otros integrantes de la escuela biotipo-

28 Bien demostrativa de la preocupación por la selección de inmigrantes es la encuesta sobre las características que debían tener los «extranjeros deseables» realizada en 1919 por el Museo Social Argentino, institución creada en 1911 y convertida en el mas influyente grupo de presión sobre todos los gobiernos sucedidos hasta la llegada del peronismo.

29 Véase MiRANDA, M., op. cit. 
lógica genovesa como el sacerdote Barbara, Vidoni, Bufano y Antognetti, ampliaron desde un principio el staff de miembros honorarios corresponsales ${ }^{30}$.

Poco después Rossi creó la Escuela de Biotipología que tuvo un área troncal en la medicina del trabajo que dirigió Donato Boccia, bajo lineamientos pendeanos que reconocían a su vez las mediaciones de la psicotecnia tomista del padre Gemelli.

Y si en los Anales de la Asociación Argentina de Biotipología, Eugenesia y Medicina Social podía encontrarse información sorprendentemente actualizada de las leyes raciales alemanas, era la «biología política» italiana la ciencia que mayor interés despertaba. La red que Pende pretendía propagar y que el fascismo asimiló a su estrategia de expansión imperial de la raza latina, ya tenía claramente un eslabón en la Argentina, en tanto que tras la guerra civil se consolidó otro en España. Precisamente la contienda había puesto de relieve no solo la colaboración militar de Italia con las fuerzas del general Francisco Franco sino también la solidaridad expresa que ese bando recibió de la Asociación Argentina de Biotipología, Eugenesia y Medicina Social. De hecho, la figura de José Antonio Primo de Rivera, líder del falangismo español que buscó extender a ese país los alcances del fascismo, y pronto convertido en el «primer mártir» del franquismo tras ser fusilado en Alicante antes de estallar la guerra civil, contribuyó a acentuar los vínculos de la red eugénica en el mundo latino. Los biotipólogos argentinos e italianos se aunaron en homenajearlo, remarcando que había pasado sus últimos días acompañado de un libro de Pende del que dejó una traducción en español «a fin de divulgarla entre los que cultivan los estudios biotipológicos» ${ }^{31}$.

Sobre la base de esta común filiación biopolítica, Rossi podía destacar que España e Italia poseían las razas «biológicamente superiores» y a partir de allí reclamar enfáticamente a las autoridades argentinas que sostengan «a cualquier precio y por todos los medios la configuración hispánico-latina de nuestro pueblo» compuesto de «hogares cristianos»y familias bien constituidas ${ }^{32}$.

Pero todas estas enormes repercusiones que la biotipológía tenía en el mundo latino y la red eugénica que había contribuido a conformar desde el papel prota-

30 Sobre el surgimiento y desarrollo de las instituciones eugénicas en Argentina véase MirandA, M. y VALLEJO G. (2004), «Las huellas de Galton: Eugenesia y control social en la Argentina del siglo XX», Taller, $\mathrm{n}^{\circ}$ 21, Buenos Aires.

31 «La obra de Nicola Pende y el Dr. J. A. Primo de Rivera»; Anales de la Asociación Argentina de Biotipología, Eugenesia y Medicina Social, nº 75, Buenos Aires, 1937.

32 Rossi, A. (1941), «Herencia, constitución, eugenesia y ortogénesis. Estudio biológicojurídico-social (cont.)» Anales de Biotipología, Eugenesia y Medicina Social, nº 96, Buenos Aires, abril-mayo, p. 6. 
gónico de la ciencia italiana, no conformaron a Pende. Su concepción de la eugenesia como una ciencia que requería de aplicación «práctica» lo instaba a él también a acrecentar su intervención política dentro de un régimen por demás permeable a sus ideas.

Precisamente de los crecientes compromisos con el Partido Fascista Italiano provino la reorientación que le dio a la denominación de su creación científica. En Pende la biotipología que lanzara en 1922, aparecería a partir de la década siguiente inmersa en la mas amplia idea italiana de bonificación humana. La raíz de esta concepto se halla en los usos publicitarios que el fascismo le dio a la «Bonifica», esto es bonificación entendida como saneamiento en un sentido amplio. La bonifica constituyó en la Italia fascista un slogan que pobló los actos públicos y las intervenciones territoriales para invadir otras esferas políticas, hasta convertirse en un concepto cada vez más ligado con los ideales íntimos del Duce que, por ejemplo, el arquitecto Giuseppe Terragni pretendió complacer en 1932 con su proyecto de monumento para la «Bonifica Integrale» en el marco de las celebraciones del decenio de la revolución fascista ${ }^{33}$.

En ese contexto Pende relanzó su doctrina publicando en 1933 la obra Bonifica umana racional y biologia politica que la dedicó a Benito Mussolini por dar con sus «principios sanos de la política biológica un hábito físico, moral e intelectual nuevo para una nueva gran Patria» ${ }^{34}$. Para Pende, Mussolini era, «mas que cualquier otro hombre antiguo y moderno, quien ha comprendido que la organización estatal no es solo un gran organismo de células-individuos, el cual debe vivir según las leyes naturales de la biología». Y la Italia del Duce era la primera nación en acoger «con simpatía el primer ensayo de esta ciencia eminentemente práctica y de pura marca italiana, que mira por una parte a la bonificación humana racional y por otra a la construcción de un Estado perfectamente armónico y robusto moral y materialmente» ${ }^{35}$.

La bonificación humana era para Pende la demostración «científica» de las directas analogía que tenía el fascismo con los fundamentos biológicos que sostenían su biotipología. Desde esta perspectiva, el paso del darwinismo social de

33 Para celebrar el aniversario de la marcha sobre Roma, en 1931 comenzaron los preparativos de la Muestra Decenal de la Revolución Fascista que dos años después se llevó a cabo con un montaje escenográfico desplegado sobre el ochocentista palacio de Exposiciones ubicado en la via Nazionale de Roma. El evento afianzó la estrecha ligazón entre el régimen y arquitectos racionalistas como Libera, De Renzi, Valente, Piacentini y el propio Terragni.

34 Pende, N. (1933), Bonifica Umana Razionale e Biologia Politica, Bolonia, Licinio Cappelli Editore, p. 5.

35 Ibidem. 
regímenes ultraliberales al fascismo, venía signado por un equivalente desplazamiento de un enfoque biológico que entendía el organismo como el marco de una competencia interindividual generalizada hacia otro de corte vitalista que lo consideraban como el resultado de interacciones corporativas.

Siendo los hombres células de un gran organismo social, a tono con el pensamiento aristotélico-tomista, Pende destacaba la necesaria supremacía del todo sobre las partes que en biología encontraba su fundamento en la ley del «altruismo celular». Ley que demostraba cómo el instinto egoísta de conservación de cada ser viviente, debía subordinarse a aquel de la asociación altruista, basado en la renuncia de la libertad de los individuos y de la cesión de los productos del propio trabajo en beneficio de la utilidad colectiva. Era ahí donde Pende encontraba el profundo arraigo biológico del gran principio del régimen fascista: «aquel en el que la libertad individual queda condicionada por la libertad y el interés colectivo» ${ }^{36}$.

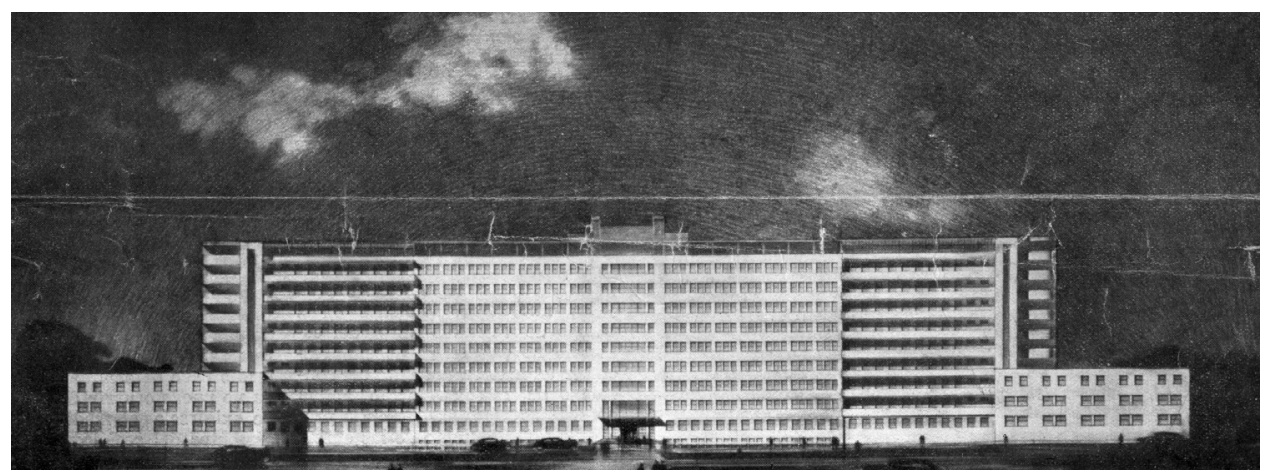

Instituto de Bonificación Humana y Ortogénesis de la Raza de Roma. Vista principal.

De este organicismo derivaba a su vez la fundamentación de las desigualdades sociales. «Del mismo modo que en la colectividad de los tejidos y de las células de un organismo existen, para la gran ley de la división del trabajo, clases celulares energéticamente diferenciadas que trabajan en armonía unas con otras en el recíproco interés, que es el interés colectivo, del mismo modo igualmente en el organismo nacional las clases de ciudadanos serán ahora antes las clases biológicas, las clases energéticamente diferenciadas de los trabajadores y productores ${ }^{37}$. Para

\footnotetext{
36 Ibidem, p. 12.

37 Ibidem, p. 38.
} 
Pende entonces la construcción de una sociedad nacional ideal era una meta que no podría plantearse, sino sobre la «diferenciación biológica y energética de los ciudadanos, y sobre las distintas corporaciones productivas». Precisamente esa división corporativa al complementarse con el rol armonizador de un Estado fuerte, «unitario y unificador», terminaba de conformar lo que Pende veía como la directa contraparte social de la biología totalizadora del individuo.

La bonificación humana de Pende fue, de este modo, el mas audaz intento de fundamentación biológica del fascismo.

\section{EL INSTITUTO PARA LA BONIFICACIÓN HUMANA Y ORTOGÉNESIS DE LA RAZA DE ROMA}

Durante el transcurso de la década de 1930 las actividades de Pende fueron enraizándose cada vez mas con el fascismo y con la centralidad histórica y cultural que el régimen italiano le confería a Roma en sus creciente vocación imperial. Las muy difundidas elucubraciones científicas de la raza itálica construidas por el creador de la biotipología se ensamblaban ahora perfectamente con el afán del Duce de hacer de Roma el epicentro de la civilidad a partir de un vasto emprendimiento. Nos estamos refiriendo a la Exposición Universal de Roma que en 1937 comenzó a prepararse para conmemorar en 1942 el veintenio del ascenso del fascismo al poder. La Muestra Decenal de la Revolución Fascista quedaría absolutamente inferiorizada - como las recientes Exposiciones Universales de Bruselas (1935) y París (1937) - por la magnitud del nuevo evento que bajo la denominación de E42 se corporizaría en una imponente intervención urbanística de 400 hectáreas llamada a orientar físicamente la expansión imperial ultramarina de la Italia mussoliniana. En efecto, la E42 indicaría con su ubicación extraurbana la proyección del núcleo monumental romano-fascista del centro antiguo hacia el mar, anticipando la conformación de una futura metrópoli que yendo de Roma a Ostia simbolizaría la recuperación de la hegemonía sobre el Mare Nostrum ${ }^{38}$.

Precisamente la llamada Via Imperiale organizaba la composición general de la E42 extendiéndose hasta la plaza Venecia y hasta Ostia para situar el nuevo conjunto edilicio en un punto intermedio del enlace de Roma con el mar. La E42, también llamada «Olimpíada de la civilidad» para ser colocada en un plano comparativo con las Olimpíadas celebradas en 1936 en Berlín, canalizaban a su vez los

38 SiCA, P. (1981), Historia del urbanismo. Siglo XX, Madrid, Instituto de Estudios de Administración Local, p. 427. 
crecientes vínculos instaurados en el plano político y cultural entre Alemania e Italia que devendrían en un escenográfico entorno arquitectónico de proporciones colosales que no ocultaban la finalidad propagandística de resaltar esos vínculos entre el fascismo y el nazismo ${ }^{39}$. Competición interimperial y voluntad de integrar las evidentes afinidades culturales confluían el notorio propósito de reproducir las valencias políticas de las olimpíadas berlinesas, donde el arquitecto Marcello Piacentini empezaba a delinear para la Roma de 1942 la contraparte «latina» de la monumental Berlín que estaba diseñando Albert Speer para $1955^{40}$.

En la medida en que las tareas organizativas de la E42 avanzaron, fue acrecentándose el peso de las cuestiones raciales en los objetivos centrales que animarían la celebración del veintenio. El año de 1938 resultó decisivo, fundamentalmente cuando de la escalada de antisemitismo del estado alemán se hizo eco el fascismo italiano que no estaba dispuesto a dejar que su nación fuera receptora de emigrados por las depuraciones raciales del Tercer Reich, como sucedería también en Argentina que poco después adoptó la misma postura cuando el área de inmigración quedó a cargo del antropólogo antisemita Santiago Peralta. En 1938 el estado italiano lanzó una fuerte campaña «concientizadora» de los problemas raciales - recordando que tras abolir las masonería restaba terminar con el judaísmo-, a la que siguió el «Manifiesto de la raza», declaración antisemita que «estudiosos fascistas» de las universidades italianas firmaron en adhesión a las directivas del Gran Consejo del Fascismo. Ese año también tuvieron lugar otros sucesos que enfatizaron esa misma orientación como fueron las muestras de la Bonificación Integral y del Mineral Italiano, contando ésta última con el pabellón de la Defensa de la Raza como uno de sus principales «atractivos».

Una de las plumas más comprometidas con la elaboración del «Manifiesto de la raza» fue precisamente la de Pende, ya convertido en un verdadero referente «científico» en materia de raza. Esta intervención pública terminó de afirmar su prestigio dentro del régimen, que luego de congratularlo con la creación de su Instituto en Génova y de nombrarlo profesor de la universidad de Roma, lo nombró Senador fascista. De aquí en más sus teorías racistas con las correspondientes aplicaciones «prácticas» tendrían aún mayor aceptación, sobre todo a partir de la

39 SCARrocchIA, S. (1999), Albert Speer e Marcello Piacentini. L'architettura del totalitarismo negli anni trenta, MiLÁN, Skira, 1999, p. 25.

40 Para la realización de la E42 se creó un en 1937 Ente autónomo que encomendó el plan a los arquitectos Pagano, Piacentini, Piccinato, Rossi y Vietti. Al año siguiente fue designado Piacentini como director general del proyecto urbanístico de la E42, quedando la mayor parte de los arquitectos racionalistas vinculados al régimen a cargo de la realización de cada uno de los edificios. 
incorporación de las advertencias «científicas» de evitar mezclas con judíos en pos de la «defensa de la raza» italiana.

Impregnando el racismo los contenidos de la E42, cuya Comisión organizadora tenía como vicepresidente a Sabato Visco, un destacado racista que incluyó en el plan al Museo de la Raza que quedó a su cargo, Pende pronto se abrió paso en el evento. En 1938 el prestigio alcanzado le permitió integrarse a las tareas de la E42, para que, dentro de ese colosal complejo, quedara a cargo de la «Muestra de la Ortogénesis Fascista de la Estirpe». La idea de esta muestra, planteada conjuntamente con la propuesta de levantar de forma permanente un segundo instituto biotipológico - ahora de Bonificación Humana-, fue lanzada por el mismo Pende al Duce y a otros miembros de la E42 en mayo de 1938, con el propósito de exhibir las acciones llevadas a cabo por el régimen en materia de defensa del niño, de la mujer, del trabajador y de la raza. El Duce se expidió favorablemente, y tras requerir sólo la sustitución de la palabra «Estirpe» por «Raza» ${ }^{41}$, exaltó la importancia que esa iniciativa tendría al «demostrar al mundo entero cuanto más modernamente científicas son las cosas que hace el régimen por la bonificación de la raza ${ }^{42}$. El Duce también satisfizo los deseos de Pende de construir uno de los edificios permanentes de la E42 para las actividades del Instituto y la Muestra - que llevaron entonces el nombre de Bonificación Humana y Ortogénesis de la Raza-. Y lo hizo atendiendo expresamente la voluntad de Pende de situar su observatorio de la personalidad humana en la colina de la E42 que orienta la expansión romana hacia el mar, donde un predio de más de 6.400 metros cuadrados quedaron comprometidos para levantar su Instituto con capacidad para doscientas cincuenta camas, y organizar en torno suyo la sección III de la E42 denominada «Ciudad de la ciencia».

La propuesta de Pende para la Muestra que tanto entusiasmó al Duce, contemplaba la división de la Muestra en 5 partes: 1) Sección doctrinaria, que ilustraba por medio de cuadros la ortogénesis de las nuevas generaciones, 2) Sección instrumental, donde se exponían las formas de indagación biométrica, psicotécnica, psicológica, química, radiológica, acompañadas de un modelo de examen biotipológico, 3) Sección documental de los resultados ortogenéticos, con una muestra fotográfica de los éxitos en las curaciones, 4) Sección de medio y cura ortogenética, consistente en una muestra que contenía la alimentación individualizada para los niños, los instrumentos de educación física infantil, y demostraciones de terapia hormonal, 5) Un film sonoro doblado en diversas lenguas para dar cuenta de las etapas evolutivas del

41 SANTARElli, N. (1987), «Mostra dell'Ortogenesi», en Gregory, T. y TARTARO A. (comp.); E42 Utopia e scenario del Regime, Roma, Cataloghi Marsilio, 1987, t. I, p. 112.

42 Cfr. GalluzzI, P. (1987), «La storia della scienza nell'E42», en Ibidem, p. 67. 
individuo ${ }^{43}$, inmerso en la permanente preocupación que Pende tenía por dejar constancia de su labor a través de películas realizadas por el instituto Luce.

Pero más allá de la importancia asignada a la Muestra, el mayor interés de Pende y del Pío Instituto Santo Spiritu que lo acompañó en su propuesta, radicaba en la posibilidad de ver prolongada la actividad iniciada en Génova con un segundo instituto biotipológico situado estratégicamente en la más emblemática manifestación del poder de la Roma fascista y en el que podía incidir en la creación de formas a la medida de sus propósitos, que quedarían así inmortalizados en la atemporal «ciudad blanca» soñada por Piacentini y el Duce.

En este sentido si el primer instituto de Pende tuvo un preciso programa que sin embargo no incidió en la formulación de características físicas autónomas que lo distinguiera de la arquitectura hospitalaria convencional, en cambio, para este segundo emprendimiento existieron precisas prefiguraciones materiales que reforzaban en un plano simbólico y funcional los fines perseguidos. En efecto, Pende requirió para su nuevo instituto el carácter de una fortaleza que debía simbolizar el concepto «verdaderamente totalitario y fascista de la defensa de la raza itálica mussoliniana». Una ciudadela protegida por cuatro torreones exteriores que reinstalaban la obsesión pitagórica que Pende tenía por ese número y que ahora venían a connotar «los pilares basales sobre los cuales debe apoyarse la bonificación humana (tutela y preparación armónica de la mujer, tutela y preparación armónica de los trabajadores, prolificidad de la raza, conservación de la pureza)» ${ }^{44}$.

Para satisfacer al destinatario los proyectistas, Luigi y Gaspare Lenzi y Dagoberto Ortensi, debieron realizar tres propuestas. De ellas fue elegida la que más explícitamente reflejaba el carácter buscado por el padre de la biotipología en detrimento de una mayor modernidad que hubiera puesto en riesgo la representación de sus valores. Esto es, aquella que con mayor énfasis exaltaba signos de la arquitectura militar en la conformación de una nueva tipología, del mismo modo que lo hiciera el panóptico integrando elementos de protección y clausura tan antiguos como un torreón rodeado de murallas, aunque paradójicamente con ellos se buscara crear un «espacio de legibilidad detallada» ${ }^{45}$.

La propuesta finalmente materializada, permitía entender que el edificio poseía fuertes particularidades: no era «un Hospital ni una Clínica ni un instituto de estudios científicos» sino la expresión de «una directiva totalmente nueva» para

43 SANTARELli, N. (1987), p. 112.

44 Noccioli, M. «Instituto dell'Ortogenesi» (pp. 506-508), en CAlvesi, M., GuidONI E. y LuX, S. (comp.) (1987), E42 Utopia e scenario del Regime, Roma, Cataloghi Marsilio, p. 508.

45 Foucault, M. (1979), p. 17. 
dar respuesta a una «obra social del Régimen verdaderamente nueva, original y puramente fascista como es la Bonificación racional de la raza» ${ }^{46}$.

El instituto era una fortaleza «asediada» con forma de hexaedro en cuyos vértices del edificio principal se levantan los cuatro torreones enmarcando el portal de honor de acceso y el amplio hall presidido por el busto del Papa Pío XII. Esos bastiones de la ciudadela contenían las circulaciones verticales que al expresarse exteriormente para completar las analogías simbólicas buscadas por Pende, determinaban funcionalmente la ubicación de las circulaciones horizontales hacia al frente y la de todas las habitaciones hacia el interior del conjunto.

Y llegando entonces mucho mas allá de la lógica parlante de signos exteriores para connotar con las formas el sentido mismo de las actividades que tendrían lugar en su interior, el punto jerarquizado de toda la composición, aquel al que confluían todas las miradas y tensiones, como lo era el torreón de vigilancia del panóptico de Bentham, lo ocupaba la capilla en el instituto de Pende.

Un NUEVo InSTITUTO DE PENDE Y LA CONTINUIDAD DE LA BIOTIPOLOGÍA EN ARGENTINA

Como sucediera con el lanzamiento del primer instituto de Pende, tampoco el del segundo pasó inadvertido en Argentina. Sobre la base de su programa Arturo Rossi impulsó en 1939 un ambiciosa reestructuración de las entidades eugénicas de ese país que colocó a la antes mencionada Asociación Argentina de Biotipología, Eugenesia y Medicina Social y la Escuela de Biotipología que estaba bajo su órbita dentro de un organismo unificado: el Instituto Nacional de Biotipología y Medicina del Trabajo.

La propuesta de Rossi fue enunciada en el «Primer Congreso Argentino de Sociología y Medicina del Trabajo» organizado en Buenos Aires por la Escuela de Biotipología que estaba a su cargo. Allí el principal interlocutor de Pende en la Argentina, recurrió a los más remanidos latiguillos «científicos» de la biotipología, para justificar la necesidad de levantar una «clínica para sanos», un laboratorio escrutador de aquellas «debilidades y errores de la constitución del cuerpo y la conformación del alma» que la normalidad esconde y que «existen en estado latente en la totalidad de los sujetos considerados aparentemente sanos». Aún de aquellos que impregnados de una recurrente apelación a las analogías con la guerra inducían a pensar el Instituto Biotipológico como un verdadero cuartel general para

46 Noccioli, M. (1987), p. 507. 
la detección de los «enemigos físicos o psíquicos que en estado de acecho amenazan permanentemente la salud física, espiritual y moral del organismo» ${ }^{47}$.

El Instituto Nacional de Biotipología y Medicina del Trabajo tuvo también un proyecto arquitectónico acorde a los fines perseguidos que realizaron los arquitectos vanguardistas Onetto, Ugarte y Ballvé Cañás. Consistía en un complejo desarrollado en dos manzanas, con pabellones articulados de tres niveles y un edificio central de ciento cincuenta metros de longitud y nueve niveles, donde la metáfora pendeana del observatorio de todos los comportamientos humanos en una fortaleza inexpugnable situada en lo alto de una colina, se redefinía en clave modernista para devenir en un gran bloque enclavado en el corazón de la metrópolis argentina.

Y aunque el edificio proyectado no se construyera, el Instituto en sí fue creado y nacionalizado en 1943 cuando era secretario de salud el médico militar de larga actuación en el eugenismo argentino, Eugenio Galli. Al año siguiente otro eugenista, Gustavo Martínez Zubiría, siendo ministro de educación convirtió la Escuela de Biotipología en el Instituto Nacional de Biotipología y Materias Afines, afirmando la principal orientación del establecimiento que fue la de formar biotipólogos. La constante reactualización de los planteos básicos de la biotipología italiana quedaron plasmados en obras de sus profesores que definían prototipos raciales actualizando y generalmente acrecentando las restricciones establecidas para contraer matrimonio y tener descendencia planteadas por Pende, que de por si trascendían las implementadas por el tercer Reich ${ }^{48}$. De ellas se destacan los tres voluminosos tomos del Tratado teórico práctico de Biotipología y Ortogénesis de Arturo Rossi, y la traducción de obras de Pende al español, prosiguiendo los desvelos del falangista Primo de Rivera, empezando por la versión de Donato Boccia y Arturo Rossi del Tratado de Biotipología Humana, Individual y Social con aplicaciones a la medicina preventiva, a la clínica, a la política biológica y a la sociología escrita por Pende veinticinco años antes.

En 1947 ya eran tantos los egresados del Instituto Nacional de Biotipología y Materias Afines que el entonces secretario de salud del primer gobierno peronista, Ramón Carrillo, también eugenista, debió intervenir para zanjar el problema

47 Rossi, A. (1940), «Instituto de Biotipología y Medicina del Trabajo», Anales de Biotipología, Eugenesia y Medicina Social, no 89, Buenos Aires, enero, p. 21.

48 En esta sintonía se hallan las obras española que buscaban colocar la biotipología a tono con la nueva realidad política de ese país. En 1941 Leopoldo Mompó Aliño publicó en Barcelona, Biotipología: estudio total de la personalidad humana con sus correlaciones morfológicas, temperamentales, caracteriológicas e intelectivas, y en 1947 el principal referente de la psiquiatría franquista, Antonio Vallejo Nágera, publicó su Biotipología. 
de incumbencias con la corporación médica a través de la preparación de un decreto presidencial que dispuso que los biotipólogos podían trabajar sin control de los médicos cuando se dedicaran únicamente a «examinar a los sanos» ${ }^{49}$.

Durante el primer y segundo gobierno de Perón (1946-1955), la biotipología italiana tuvo un amplio campo de acción con algunos matices como los que Carrillo introdujo en el Instituto Nacional de Biotipología y Materias Afines, para dotarlo de un mayor sesgo nacionalista a tono con su propia trayectoria personal en el ejército y de contenidos provenientes de la experiencia eugénica del francés Alexis Carrel y el Instituto del Hombre que creó en Vichy bajo la ocupación alemana. El resultado sería la creación del Instituto Argentino del Hombre.

El largo recorrido de la biotipología en Argentina no se interrumpió con la caída del peronismo. La reacción antiperonista que se impuso militarmente en 1955 abrió nuevos espacios para su desarrollo, especialmente cuando el radical Bernaldo de Quirós, después de haber creado la Sociedad Argentina de Eugenesia que tuvo las explícitas colaboraciones de Ricardo Balbín y Arturo Frondizi ${ }^{50}$, montó en 1957 la Facultad de Eugenesia dentro de la Universidad del Museo Social Argentino que fue subsidiada ininterrumpidamente por el estado hasta 1973. Con la biotipología como materia troncal, se formaron por mas de dos décadas profesionales preparados para realizar, entre otro tipo de atenciones psicológico-confesionales, consultas prematrimoniales de corte eugénico que fijaban las características físicas y temperamentales que debía buscar un cónyuge en el otro por el bien de la descendencia.

Para entonces la ciencia de la personalidad humana se había difuminado en múltiples esferas demostrando que si la utopía pendeana de crear una red de grandes establecimientos especialmente preparados para detectar lo «anormal» en los «aparentemente sanos» se desplomó con el régimen que la sostenía, no sucedió lo mismo Argentina con sus objetivos.

La sorprendente continuidad de una ciencia del control social que durante más de cuatro décadas implementó fichas biotipológicas en las áreas de minoridad, educación, medicina del trabajo, y fuerzas armadas, para medir, clasificar, seleccionar y disciplinar, la infancia abandonada, adolescentes, obreros y conscriptos y soldados, no fue desaprovechada por la última dictadura militar que agregó a esa lista la obsesiva detección de subversivos.

49 Decreto del Poder Ejecutivo Nacional no 16.187, del 2 de agosto de 1950.

50 Balbín fue durante años el principal líder de la oposición al peronismo en tanto que Frondizi fue Presidente de la Nación entre 1958 y 1962. 